\title{
A 2-DIMENSIONAL MAGNETOSTATIC MODEL OF THE BOOSTER RING QUADRUPOLE MAGNETS
}

BOOSTER TECHNICAL NOTE NO. 177

\author{
M. A. GOLDMAN \\ SEPTEMBER 20, 1990
}

ALTERNATING GRADIENT SYNCHROTRON DEPARTMENT BROOKHAVEN NATIONAL LABORATORY UPTON, NEW YORK 11973 


\title{
A 2-Dimensional Magnetostatic Model
}

\section{Of The}

\author{
Booster Ring Quadrupole Magnets
}

M.A. Goldman

August 14, 1990

\begin{abstract}
In order to document some aspects of the Booster quadrupole magnet design, the magnetic fields, field gradients, and multi-pole coefficients of the magnetic field were calculated.

From these modelling computations, carried out for a magnet of perfect constructional symmetry, the field gradient in the horizontal midplane is flat out to $8 \mathrm{~cm}$ from the magnetic axis, for currents up to 5700 Amperes. The ratio of dodecapole to quadrupole harmonic coefficients decreases with current in this range. The magnitude of the saturation, at 5700 Amperes current is 4 to 5 percent, depending upon the stacking fraction of the magnet laminations.
\end{abstract}

Graphs of: calculated magnetic field gradient versus position in the magnet's horizontal midplane; quadrupole coefficient, ratio of dodecapole to quadrupole coefficient, and magnetic saturation versus magnet current are given. A table of calculated multipole coefficients versus magnet current is given.

\section{Description Of The Computations}

A two-dimensional magnetostatic analysis of the Booster ring quadrupole magnets was made, using the POISSON codes. The magnetic fields, field gradients and multipole coefficients were computed assuming perfect constructional symmetry.

The analysis was performed to study the onset of saturation in the magnet, as the magnet current increases to and beyond its full operating value. A 4-fold-symmetric geometry was assumed. A second set of computations, to be made subsequently, will examine the effects of magnet shape deformations due to constructional assymetries. It is hoped that such computations will contribute to an understanding of the observed magnet characteristics.

The magnet dimensions, including pole face profile, are given in BNL Booster quadrupole magnet construction drawings D36-M-0888-4A and D36-M-0917. The permeability input table used for the computation was taken from data curves of U.S.S. type M36 24-Gage fully-processed sheet steel. The data curve supplied by the manufacturer extends to 20.8 kilogauss, at a field intensity of 1 kilooersted. This range of data is 
sufficient to allow computation of the magnet fields. However, to insure computational stability during the calculation, the B-H table must be extrapolated to higher $\mathrm{H}$ fields, and in a manner that the steel's magnetization is non-decreasing in $\mathrm{H}$ and approaches saturation magnetization at high $\mathrm{H}$ fields. The B-field was set equal to $\mathrm{H}+4 \pi \mathrm{M}_{\text {sat }}$ for $\mathrm{H} \geq 4$ $\mathrm{kOe}$, and fitted smoothly in the interval from 1 to $4 \mathrm{kOe}$. A value of $22 \mathrm{kG}$ was used for $4 \pi \mathrm{M}_{\text {sat }}$. For the range of currents used the computed fields do not depend on the detailed choice of points used in extending the B-H table.

The shape of lamination used here is that of the original design by J. Jackson and G. Danby. We note that the design by Jackson and Danby was chosen not only to minimize higher multipoles but also to allow the quadrupole magnets to track the Booster dipole magnets. The overall magnet design is much more subtle than indicated by the simplified POISSON picture presented in this note.

Minor simplifications were made in the computation. Small pin notches in the lamination arms, and water cooling spaces inside the conductors were omitted from the model. The insulation gaps between lamination arms were replaced by an increase of 0.006 $\mathrm{cm}$ in the length of each lamination quadrant arm; the computation was thereby carried out for a pole tip 2 radius of $8.2524 \mathrm{~cm}$, instead of the nominal $8.255 \mathrm{~cm}$ specified in the Booster Design Manual (but within the mechanical tolerances of the design). These changes cause only minor perturbations in the results, and their adoption appreciably simplifies setting up of the model.

The POISSON family of codes is extensively described in the LANL Accelerator Code Group User's Guide [1] and Reference Manual [2]. The design, computation and symmetries of iron-dominated quadrupole magnets are described at length in an article by Fischer [3].

The quadrupole magnet is specified by an AUTOMESH file named BRQUAD.DAT;4. Only one octant is needed to describe the magnet. Six computational regions are used: to specify the magnet's outline, laminations, bolt-holes and coils; a seventh boundary region, a line boundary region, is used to impose a Dirichlet (parallel field) boundary condition along the pole's axis of symmetry and along the outer edge of the laminations.

\section{2. $\underline{\text { Results }}$}

The calculated magnetic field configuration at 5500 Ampere current is shown in Fig. 1A. The magnet quadrant geometry is shown in Fig. 1B. The field gradient at the magnet's horizontal midplane is plotted in Fig. 2. The field gradient is nearly constant, for horizontal distances up to $8 \mathrm{~cm}$ from the magnetic axis.

The magnetic field at the center of an ideal quadrupole magnet is purely quadrupole, without higher harmonic terms. Since the pole width of the magnet as designed is limited by conductor windings, higher harmonic terms appear. The lowest term allowed by the 4-fold pole symmetry is the dodecapole. Its magnitude is a measure of the field quality, and the variation of the ratio of dodecapole to quadrupole coefficient as current changes is a 
measure of the invariance of the magnetic field shape with current. As part of the POISSON code solution of the magnet, the multipole coefficients are computed. These are listed, for several magnet currents, in Table 1.

The ratio of dodecapole to quadrupole coefficient, as a function of current, is plotted in Fig. 3. Here, we assume that the vertical magnetic field component at the horizontal midplane of the magnet is represented by the power series:

$$
\mathrm{B}_{\mathrm{y}}(\mathrm{x})=\mathrm{B}_{0}+\left(\mathrm{B}_{1}\right) \mathrm{x}+\left(\mathrm{B}_{2}\right) \mathrm{x}^{2}+(\mathrm{B} 3) \mathrm{x}^{3}+\left(\mathrm{B}_{4}\right) \mathrm{x}^{4}+\ldots,
$$

when $y=0$, and the only non-zero coefficients belong to powers of $x$ of the form $4 n+1$, where $\mathrm{n}$ is a positive integer. One measure of the departure of the magnet field from linear variation with magnet current is the saturation, defined by:

$$
\% \text { SATURATION }(\mathrm{I})=(100 \%) \times\left[1-\frac{\mathrm{B}_{1}(\mathrm{Iref}) / \mathrm{Iref}}{\mathrm{B}_{1}(\mathrm{I}) / \mathrm{I}}\right]
$$

where $B_{1}(I)$ is the quadrupole harmonic coefficient at magnet current $I$, and the reference current, Iref, is chosen to be low enough that no part of the magnet core is in saturation. We choose our reference current to be 2500 Amperes. The fields in the core, at this current, are well below saturation, as shown by POISSON calculations. The value of percent saturation versus current is listed in Table 1, and is plotted in Fig. 5.

Maps of the magnetic field amplitude at selected regions of the laminated magnet core are also given. As a guide to reading these maps, an outline drawing of a magnet octant is provided (Fig. 4), giving logical coordinates at characteristic magnet positions. One notices that the steel approaches saturation locally, at the pole corners, as current increases to the upper end of its operating range. This leads to falloff of the ratio of dodecapole to quadrupole coefficients as current increases.

\section{Summary}

The Booster ring quadrupole magnets, as designed by J. Jackson and G. Danby, should have a uniform quadrupole field gradient across the midplane horizontal aperture of the magnet out to $\pm 8 \mathrm{~cm}$ of the magnetic axis, for currents up to 5700 Amperes.

The ratio of dodecapole to quadrupole coefficient decreases with increasing current. The computed multipole coefficients are listed in Table 1.

The percentage of saturation was calculated assuming lamination stacking fractions of $97 \%$ and $100 \%$. For currents up to 5700 Amperes, the calculated percentage saturation was between 0 and -5 percent. 


\section{Acknowledgements}

I wish to thank John Jackson and Gordon Danby for helpful comments relating to their design of the magnets.

I am indebted to Roy Thern for a critical discussion of the need for a properly extended permeability table in insuring the computational stability of the POISSON codes when operating near saturation, and to Ed Bleser for general discussions on multipole moments.

\section{References}

1. Los Alamos Accelerator Code Group. User's Guide For The Poisson/Superfish Group Of Codes. Los Alamos National Laboratory Report LA-UR-87-115, Los Alamos NM 87545, Jan. 1987.

2. Ibid. Reference Manual For The Poisson/Superfish Group Of Codes. LAUR-87-126. Jan 1, 1987.

3. G.E. Fisher, Iron Dominated Magnets. Article in AIP Conference Proceedings No. 153, M. Month and M. Dienes editors, American Institute of Physics, NY, 1987. 


\section{TABLE 1}

\section{B-Field Multipole Expansions}

We list computed multipole coefficients of the series:

(1) $\left(B_{y}+j B_{x}\right)=\underset{n}{\operatorname{SUM}}\left\{\left(B_{n}+j A_{n}\right)(\cos n \theta+j \sin n \Theta)(r / 1 \mathrm{~cm})^{* *} n\right\}$,

where the field components $\mathrm{B}_{\mathrm{X}}$ and $\mathrm{B}_{\mathrm{y}}$ are in Gauss.

For a symmetric quadrupole, all $A_{n}$ vanish, and non-zero $B_{n}$ are of the form $4 n+1$ $(n=0,1,2, \ldots)$.

In the horizontal midplane of the magnet $(\theta=0, r=x)$ the series reduces to:

(2) $\mathrm{B}_{\mathrm{y}}(\mathrm{x}, \mathrm{y}=0)=\mathrm{B}_{1} \mathrm{x}+\mathrm{B}_{5} \mathrm{x}^{5}+\mathrm{B}_{9} \mathrm{x}^{9}+\mathrm{B}_{13} \mathrm{x}^{13}+\ldots$.

\begin{tabular}{|c|c|c|c|c|c|}
\hline $\begin{array}{l}\text { Magnet } \\
\text { Current } \\
\text { (Ampere) }\end{array}$ & $\mathrm{n}$ & $\begin{array}{r}\left|B_{1}\right| \\
\text { (Gauss) }\end{array}$ & $\begin{array}{l}\text { Lamination } \\
\text { Stacking } \\
\text { Fraction }\end{array}$ & $\mathrm{B}_{\mathrm{n}} / \mathrm{B}_{1}$ & \%Saturation \\
\hline 0200 & $\begin{array}{l}1 \\
5 \\
9 \\
13\end{array}$ & 036.657 & 1.00 & $\begin{array}{l}1.0000 \\
4.5415 \mathrm{E}-07 \\
1.0105 \mathrm{E}-10 \\
-1.8408 \mathrm{E}-14\end{array}$ & \\
\hline 0600 & $\begin{array}{l}1 \\
5 \\
9 \\
13\end{array}$ & 110.244 & 1.00 & $\begin{array}{l}1.0000 \\
4.6189 \mathrm{E}-07 \\
1.0099 \mathrm{E}-10 \\
-1.8405 \mathrm{E}-14\end{array}$ & \\
\hline 1100 & $\begin{array}{l}1 \\
5 \\
9 \\
13\end{array}$ & 202.300 & 1.00 & $\begin{array}{l}1.0000 \\
4.5906 \mathrm{E}-07 \\
1.0135 \mathrm{E}-10 \\
-1.8434 \mathrm{E}-14\end{array}$ & \\
\hline 1500 & $\begin{array}{l}1 \\
5 \\
9 \\
13\end{array}$ & 275.968 & 1.00 & $\begin{array}{l}1.0000 \\
4.6035 \mathrm{E}-07 \\
1.0138 \mathrm{E}-10 \\
-1.8447 \mathrm{E}-14\end{array}$ & \\
\hline
\end{tabular}




\begin{tabular}{|c|c|c|c|c|c|}
\hline 2500 & $\begin{array}{l}1 \\
5 \\
9 \\
13\end{array}$ & 460.040 & 1.00 & $\begin{array}{l}1.0000 \\
4.5655 \mathrm{E}-07 \\
1.0131 \mathrm{E}-10 \\
-1.8428 \mathrm{E}-14\end{array}$ & 0.0000 \\
\hline 3500 & $\begin{array}{l}1 \\
5 \\
9 \\
13\end{array}$ & 643.690 & 1.00 & $\begin{array}{l}1.0000 \\
4.5390 \mathrm{E}-07 \\
1.0132 \mathrm{E}-10 \\
-1.8428 \mathrm{E}-14\end{array}$ & -0.0576 \\
\hline 4250 & $\begin{array}{l}1 \\
5 \\
9 \\
13\end{array}$ & 779.459 & 1.00 & $\begin{array}{l}1.0000 \\
4.4612 \mathrm{E}-07 \\
1.0040 \mathrm{E}-10 \\
-1.8471 \mathrm{E}-14\end{array}$ & -0.3347 \\
\hline 4750 & $\begin{array}{l}1 \\
5 \\
9 \\
13\end{array}$ & 864.321 & 1.00 & $\begin{array}{l}1.0000 \\
4.3089 \mathrm{E}-07 \\
9.9495 \mathrm{E}-11 \\
-1.8529 \mathrm{E}-14\end{array}$ & -1.129 \\
\hline 5000 & $\begin{array}{l}1 \\
5 \\
9 \\
13\end{array}$ & 902.465 & 1.00 & $\begin{array}{l}1.0000 \\
4.2302 \mathrm{E}-07 \\
9.7507 \mathrm{E}-11 \\
-1.8527 \mathrm{E}-14\end{array}$ & -1.952 \\
\hline 5250 & $\begin{array}{l}1 \\
5 \\
9 \\
13\end{array}$ & 940.084 & 1.00 & $\begin{array}{l}1.0000 \\
4.0926 \mathrm{E}-07 \\
9.5817 \mathrm{E}-11 \\
-1.8534 \mathrm{E}-14\end{array}$ & -2.768 \\
\hline 5500 & $\begin{array}{l}1 \\
5 \\
9 \\
13\end{array}$ & 977.638 & 1.00 & $\begin{array}{l}1.0000 \\
3.9118 \mathrm{E}-07 \\
9.3494 \mathrm{E}-11 \\
-1.8575 \mathrm{E}-14\end{array}$ & -3.524 \\
\hline 5700 & $\begin{array}{l}1 \\
5 \\
9 \\
13\end{array}$ & 1007.14 & 1.00 & $\begin{array}{l}1.0000 \\
3.6435 \mathrm{E}-07 \\
8.9873 \mathrm{E}-11 \\
-1.8634 \mathrm{E}-14\end{array}$ & -4.146 \\
\hline 2500 & $\begin{array}{l}1 \\
5 \\
9 \\
13\end{array}$ & 460.001 & 0.97 & $\begin{array}{l}1.0000 \\
4.5622 \mathrm{E}-07 \\
1.0133 \mathrm{E}-10 \\
-1.8425 \mathrm{E}-14\end{array}$ & -0.000 \\
\hline
\end{tabular}




\begin{tabular}{|c|c|c|c|c|c|}
\hline 3500 & $\begin{array}{l}1 \\
5 \\
9 \\
13\end{array}$ & 643.536 & 0.97 & $\begin{array}{l}1.0000 \\
4.5335 \mathrm{E}-07 \\
1.0128 \mathrm{E}-10 \\
-1.8436 \mathrm{E}-14\end{array}$ & -0.0729 \\
\hline 4250 & $\begin{array}{l}1 \\
5 \\
9 \\
13\end{array}$ & 778.320 & 0.97 & $\begin{array}{l}1.0000 \\
4.4145 \mathrm{E}-07 \\
9.9815 \mathrm{E}-11 \\
-1.8483 \mathrm{E}-14\end{array}$ & -0.475 \\
\hline 4750 & $\begin{array}{l}1 \\
5 \\
9 \\
13\end{array}$ & 859.957 & 0.97 & $\begin{array}{l}1.0000 \\
4.2547 \mathrm{E}-07 \\
9.7806 \mathrm{E}-11 \\
-1.8527 \mathrm{E}-14\end{array}$ & -1.635 \\
\hline 5000 & $\begin{array}{l}1 \\
5 \\
9 \\
13\end{array}$ & 897.493 & 0.97 & $\begin{array}{l}1.0000 \\
4.1451 \mathrm{E}-07 \\
9.6488 \mathrm{E}-11 \\
-1.8537 \mathrm{E}-14\end{array}$ & -2.507 \\
\hline 5250 & $\begin{array}{l}1 \\
5 \\
9 \\
13\end{array}$ & 935.018 & 0.97 & $\begin{array}{l}1.0000 \\
3.9749 \mathrm{E}-07 \\
9.4322 \mathrm{E}-11 \\
-1.8546 \mathrm{E}-14\end{array}$ & -3.314 \\
\hline 5500 & $\begin{array}{l}1 \\
5 \\
9 \\
13\end{array}$ & 971.755 & 0.97 & $\begin{array}{l}1.0000 \\
3.6725 \mathrm{E}-07 \\
9.0295 \mathrm{E}-11 \\
-1.9625 \mathrm{E}-14\end{array}$ & -4.142 \\
\hline 5700 & $\begin{array}{l}1 \\
5 \\
9 \\
13\end{array}$ & 1000.39 & 0.97 & $\begin{array}{l}1.0000 \\
3.3346 \mathrm{E}-07 \\
8.5631 \mathrm{E}-11 \\
-1.8714 \mathrm{E}-14\end{array}$ & -4.839 \\
\hline
\end{tabular}




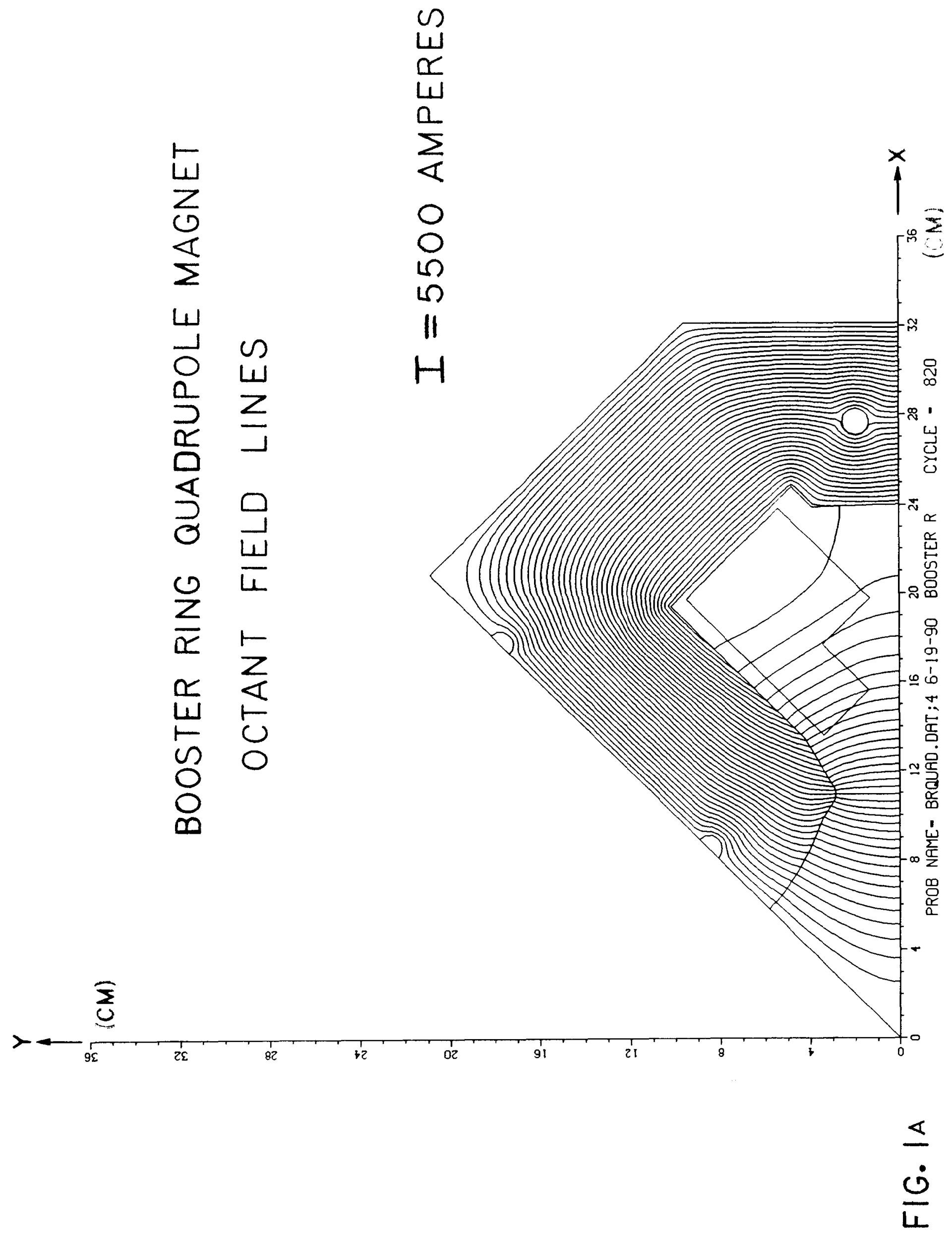




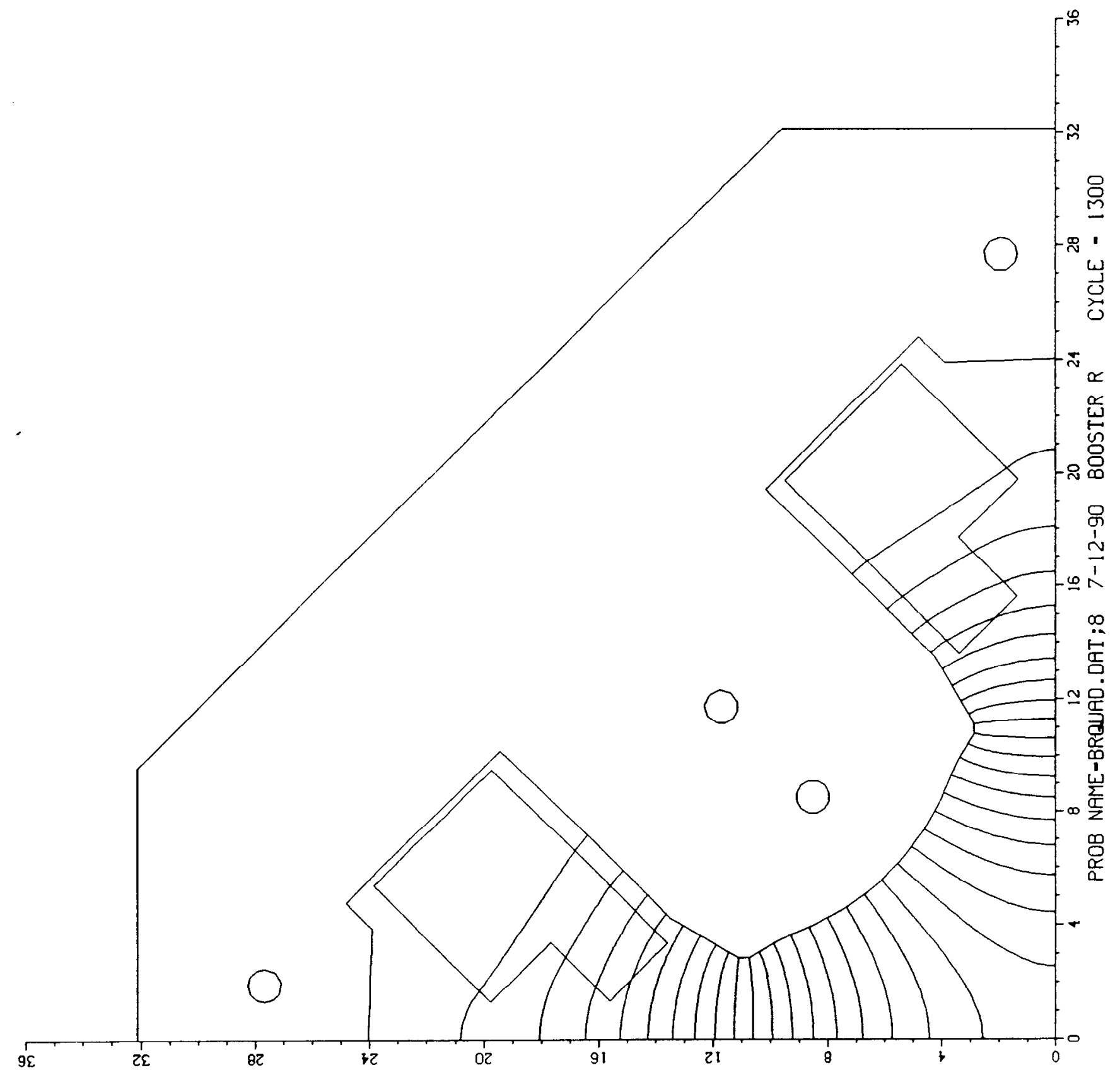

$\frac{\infty}{\dot{0}}$ 


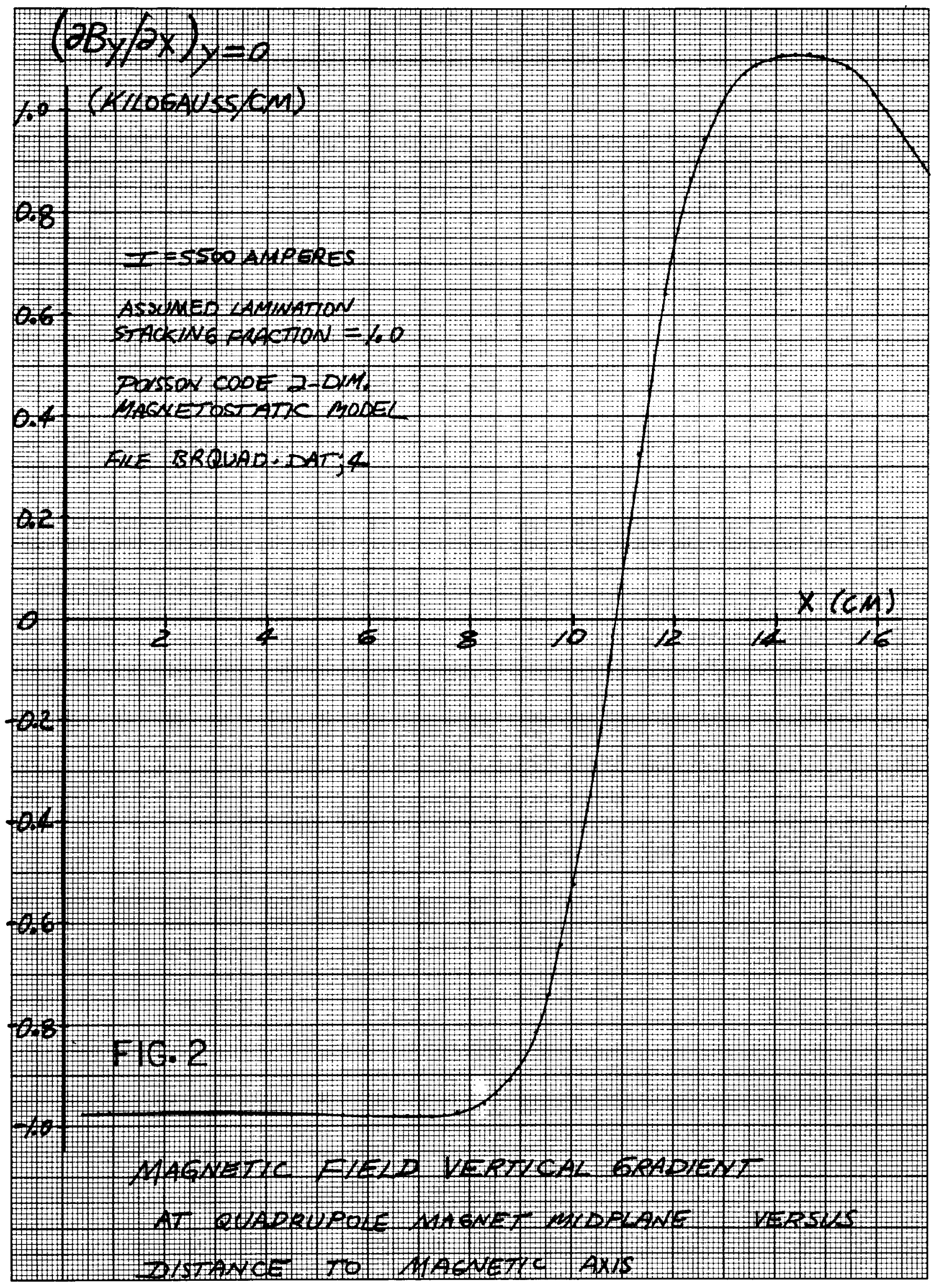




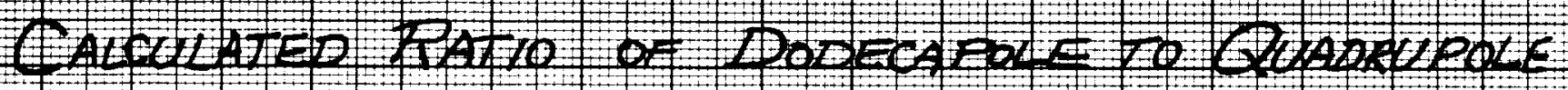
MuLrpode Coefenchents
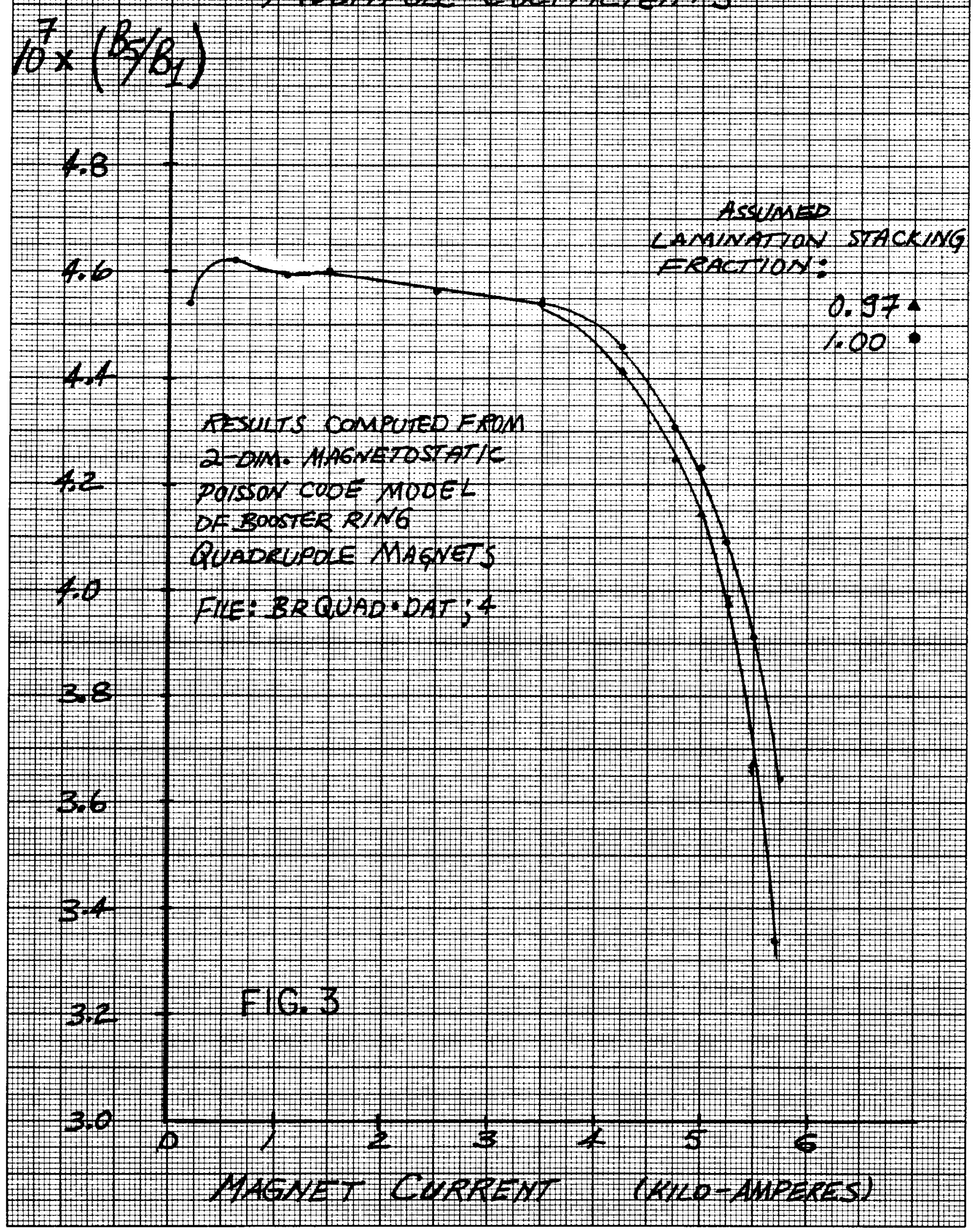


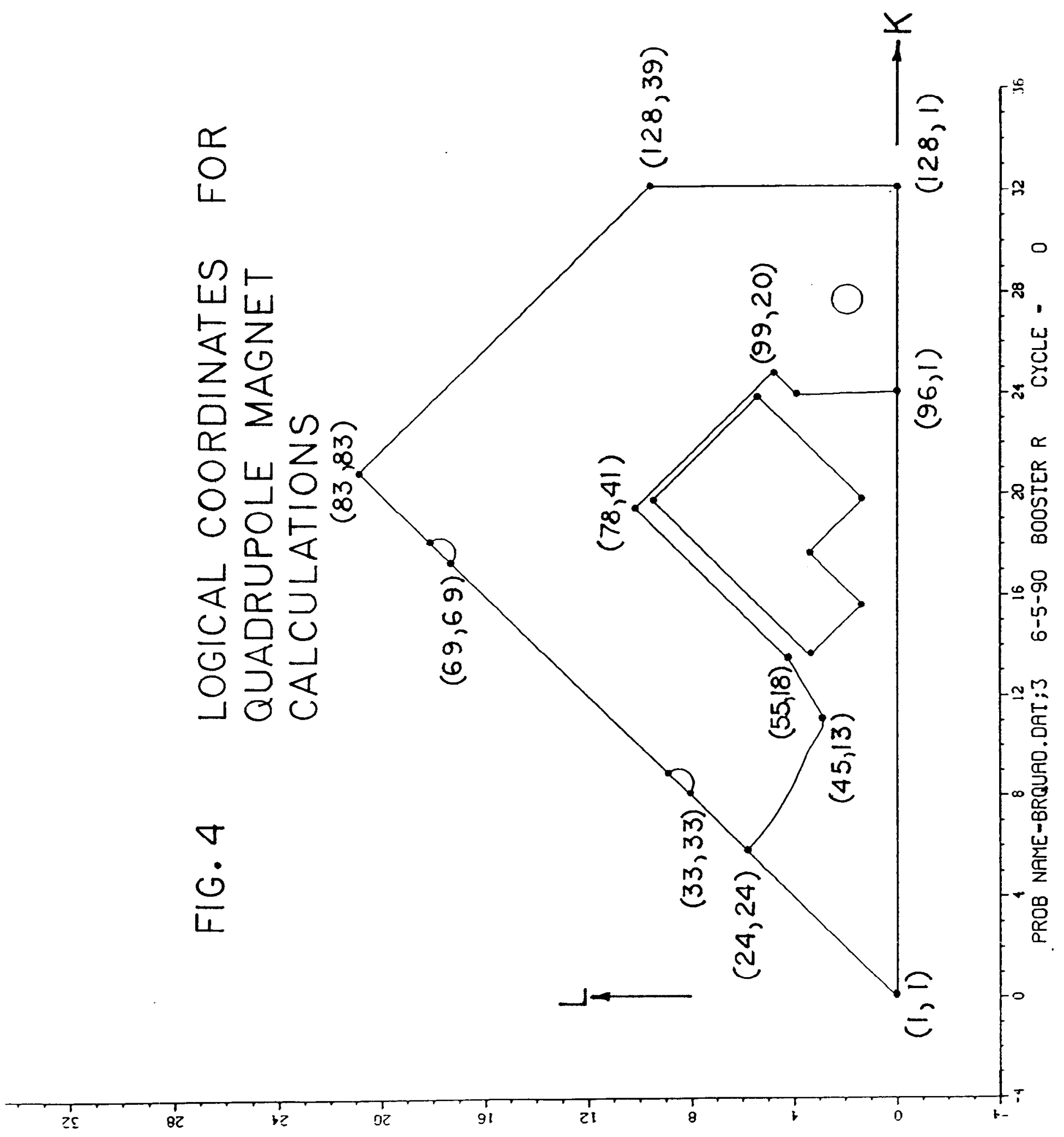


$2 x$

$\div \div$

$\rightarrow m$

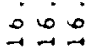

$m m n m m$

: $\dot{0}$ i

-

vmamar =

$\therefore$ : 0 :

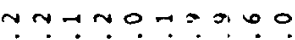

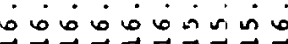

$\rightarrow 40700,000 m 0$

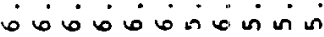

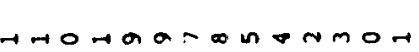

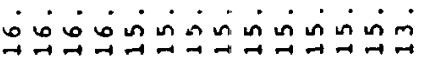

ota 0 and a th modehn

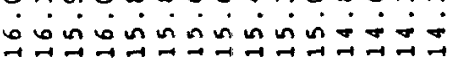

o oa a orinnmyarbann b

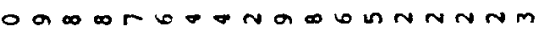

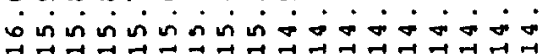

andrognamon

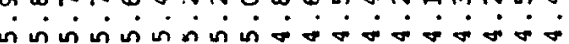

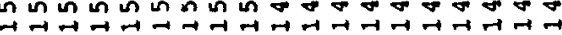

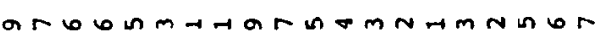

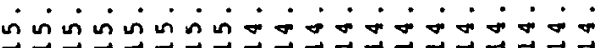

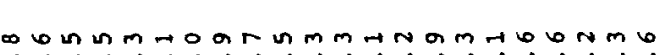

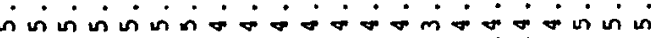

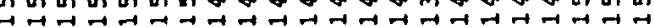

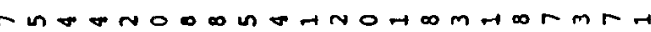

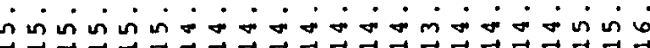

bomnom.

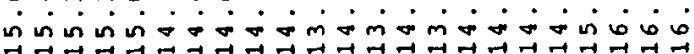

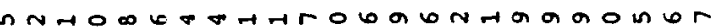

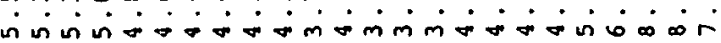

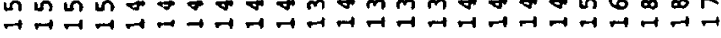

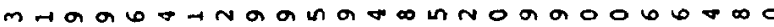

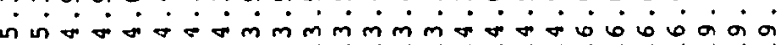

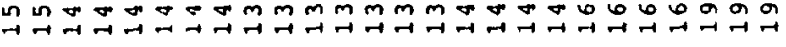

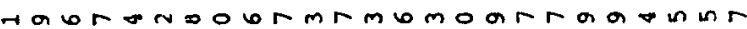

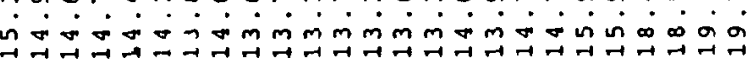

anm

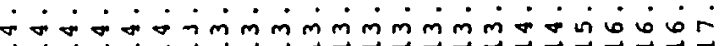

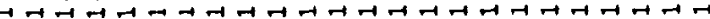

o $\rightarrow$ onmrnw

$\therefore \therefore \dot{0} \dot{m} \dot{m} \dot{m} \dot{m} \dot{m} \dot{m} \dot{m} \dot{m} \dot{m}$

$* \sigma \sigma m m m m m n m m m m m m m$ un w

ル ロ

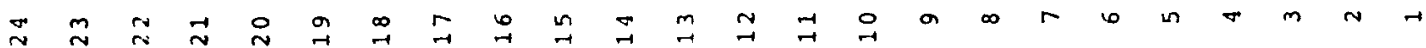




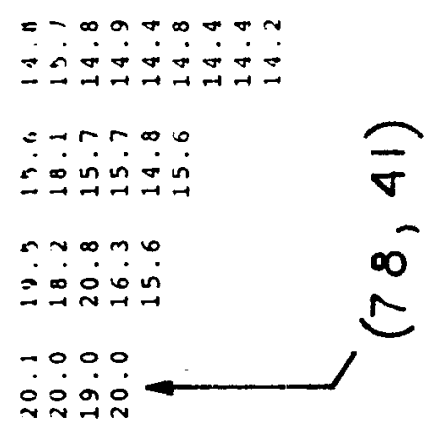

- $-0 \mathrm{mog}$

- a or a on $\infty$

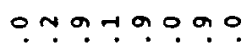

a $\dot{a}_{\rightarrow \rightarrow}^{\infty} \dot{a}_{\rightarrow \rightarrow}^{\infty}$ के

a o a o a o a a a

$\infty \dot{a} \infty \dot{0} \infty \dot{\infty} \infty \dot{\infty} \infty \dot{\infty} \infty \dot{\infty}$

a 00 a o o on $\infty$ a $\infty$ a

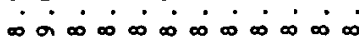

-

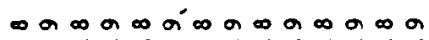

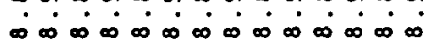

$\infty a \infty a \infty a \infty$ a $\infty$ a $\infty$ a $\infty \infty \infty$

$\dot{\infty} \dot{\infty} \dot{\infty} \dot{\infty} \dot{\infty} \dot{\infty} \dot{\infty} \dot{\infty} \dot{\infty} \dot{\infty} \dot{\infty} \dot{\infty} \dot{\infty} \dot{\infty} \dot{\infty}$

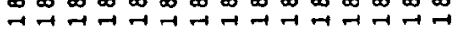

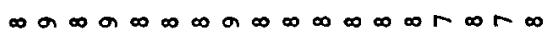

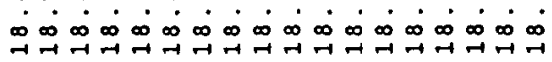

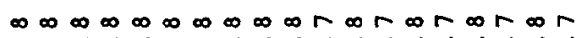

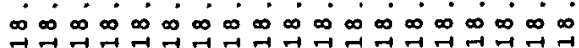

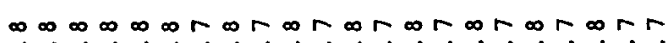

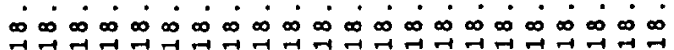

m

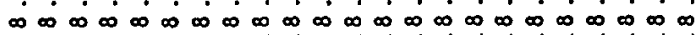

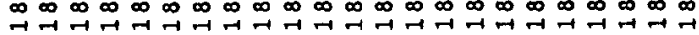

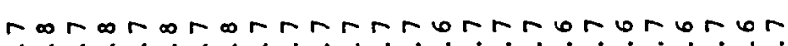

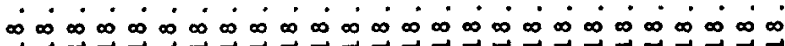

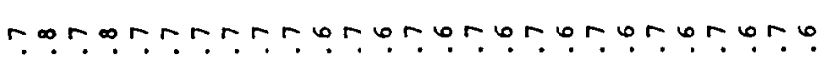

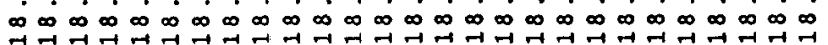

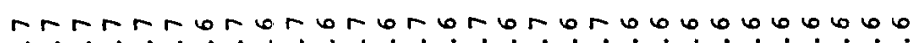

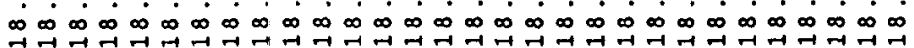

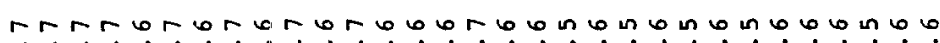
$\dot{\infty} \dot{\infty} \dot{\infty} \dot{\infty} \dot{\infty} \dot{\infty} \dot{\infty} \dot{\infty} \dot{\infty} \dot{\infty} \dot{\infty} \dot{\infty} \dot{\infty} \dot{\infty} \dot{\infty} \dot{\infty} \dot{\infty} \dot{\infty} \dot{\infty} \dot{\infty} \dot{\infty} \dot{\infty} \dot{\infty} \dot{\infty} \dot{\infty} \dot{\infty} \dot{\infty} \dot{\infty} \infty \dot{\infty} \infty$ H

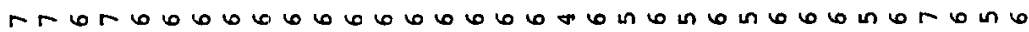

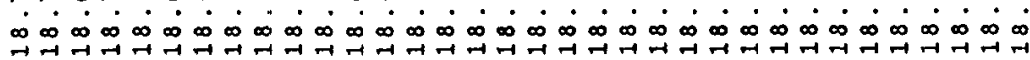

$\measuredangle$
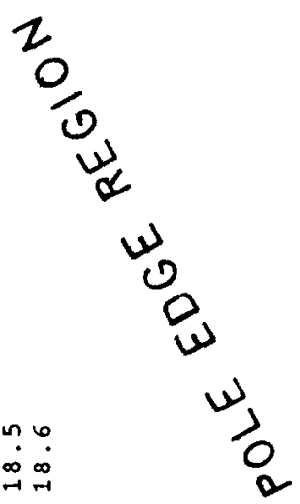

6 6 n

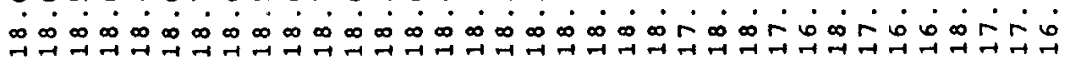

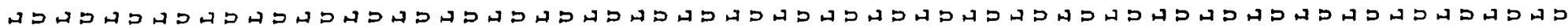

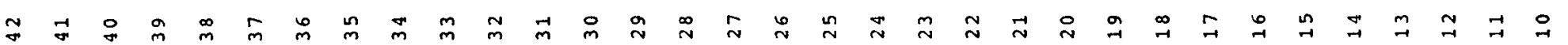


ㄴ? ?

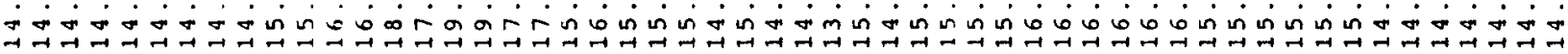

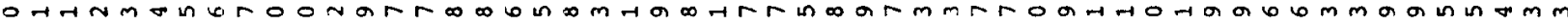

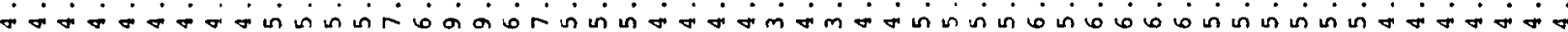
-

0 atwamarmbadabr

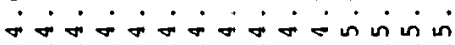

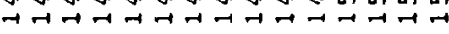

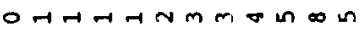

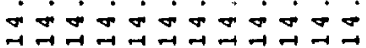

$000 h 4 h 4 n m a n$

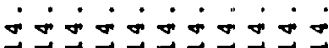

$0000 h-4 \pi-140$

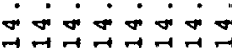

00000.7 .7

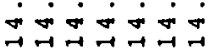

0000

ॠேंष्ष

000

$\dot{\infty}_{\rightarrow+\infty}^{\infty}$

क

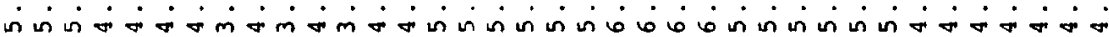

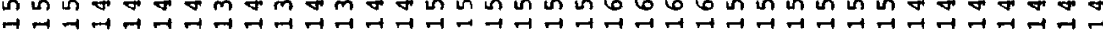

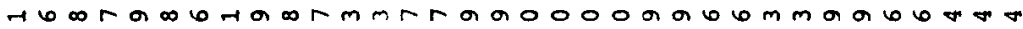

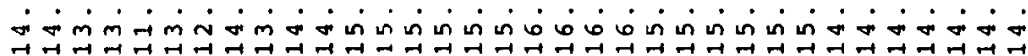

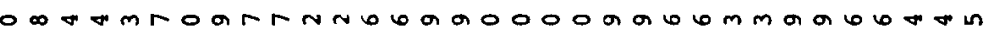

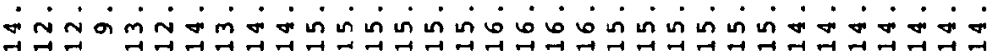

N

の

o)

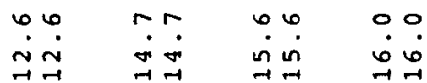

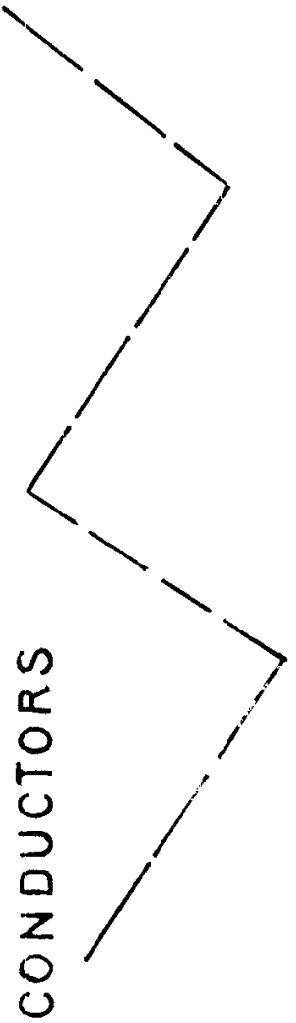

山ロ

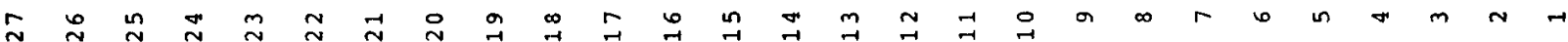




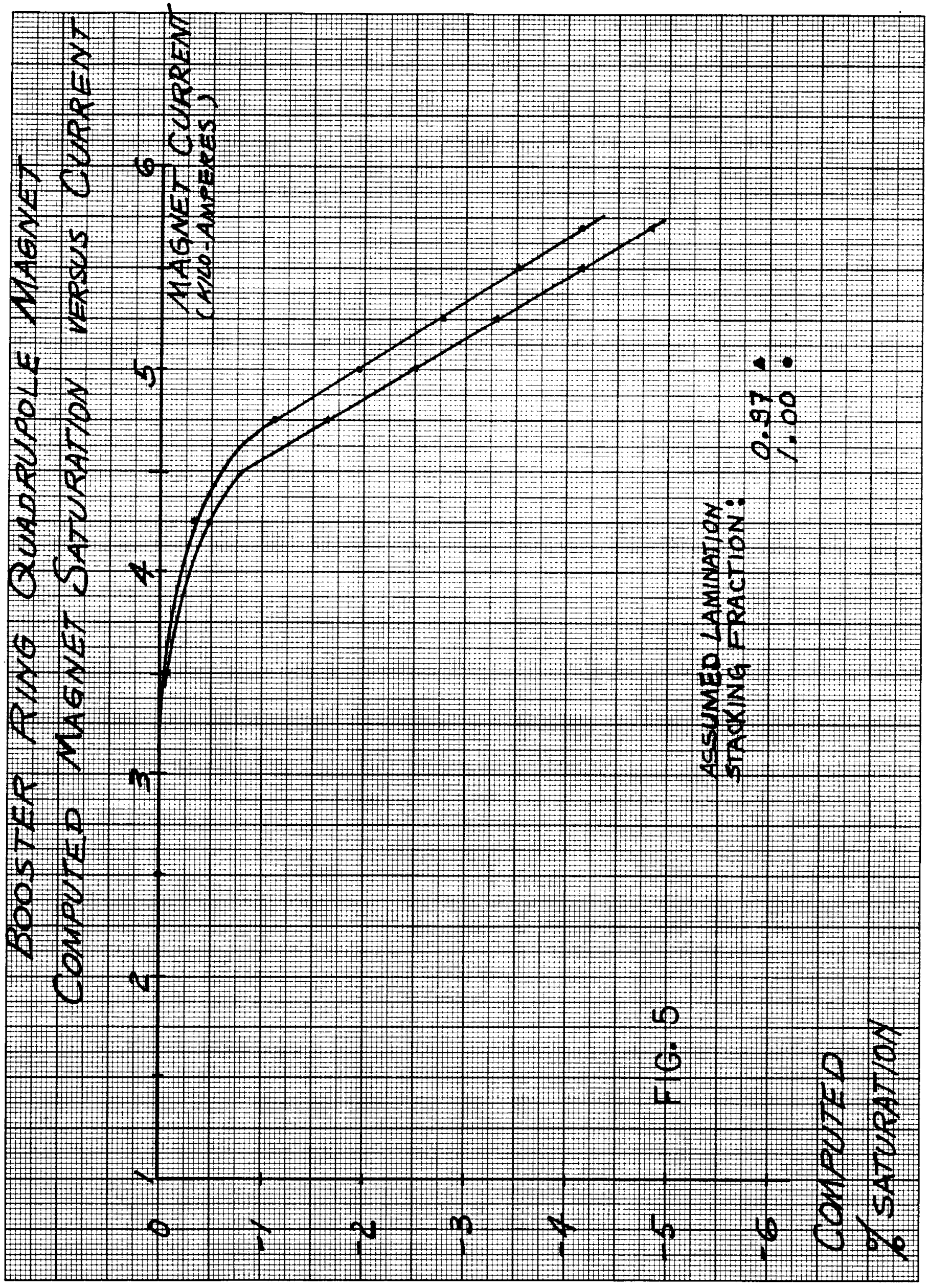




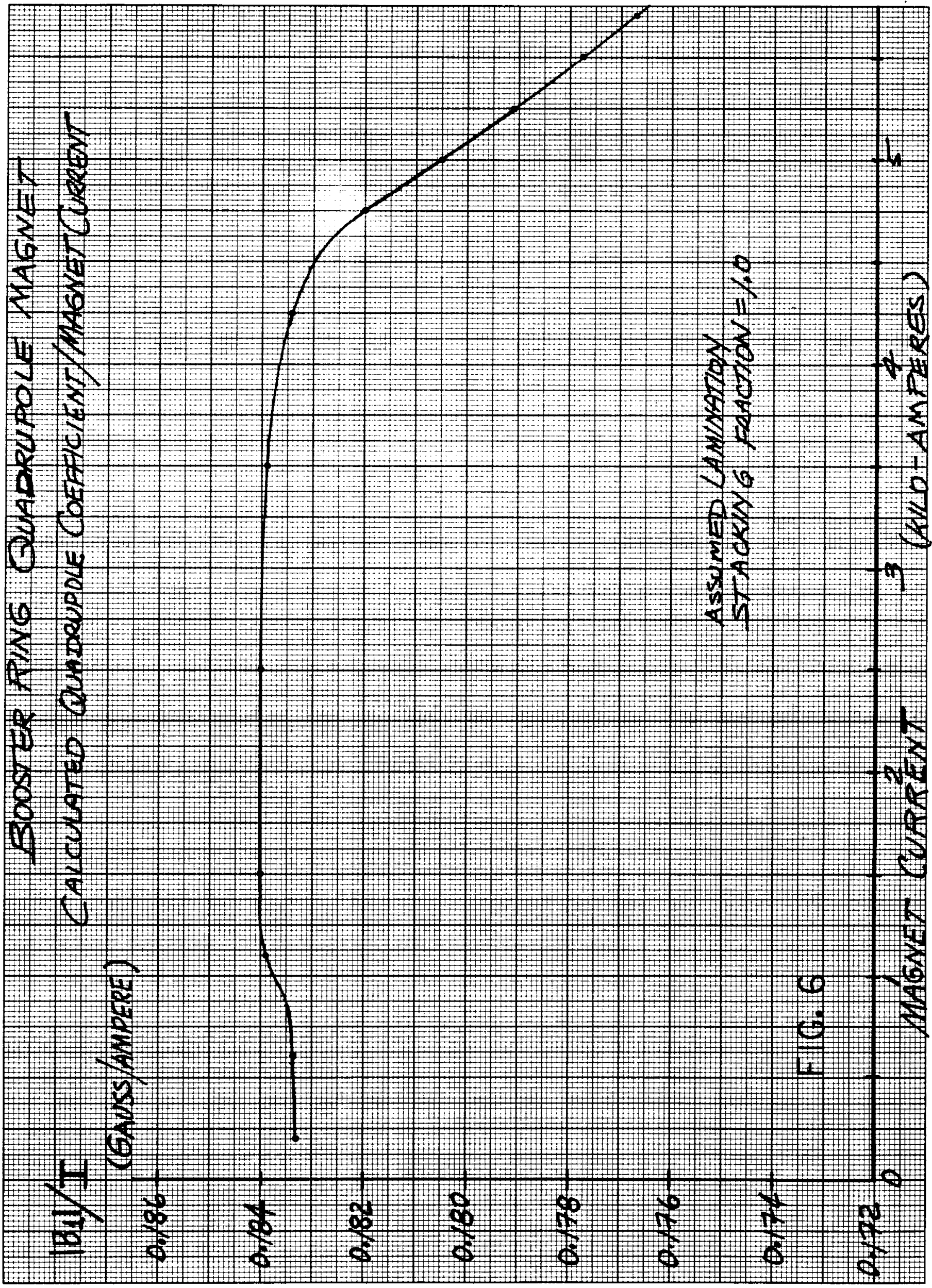



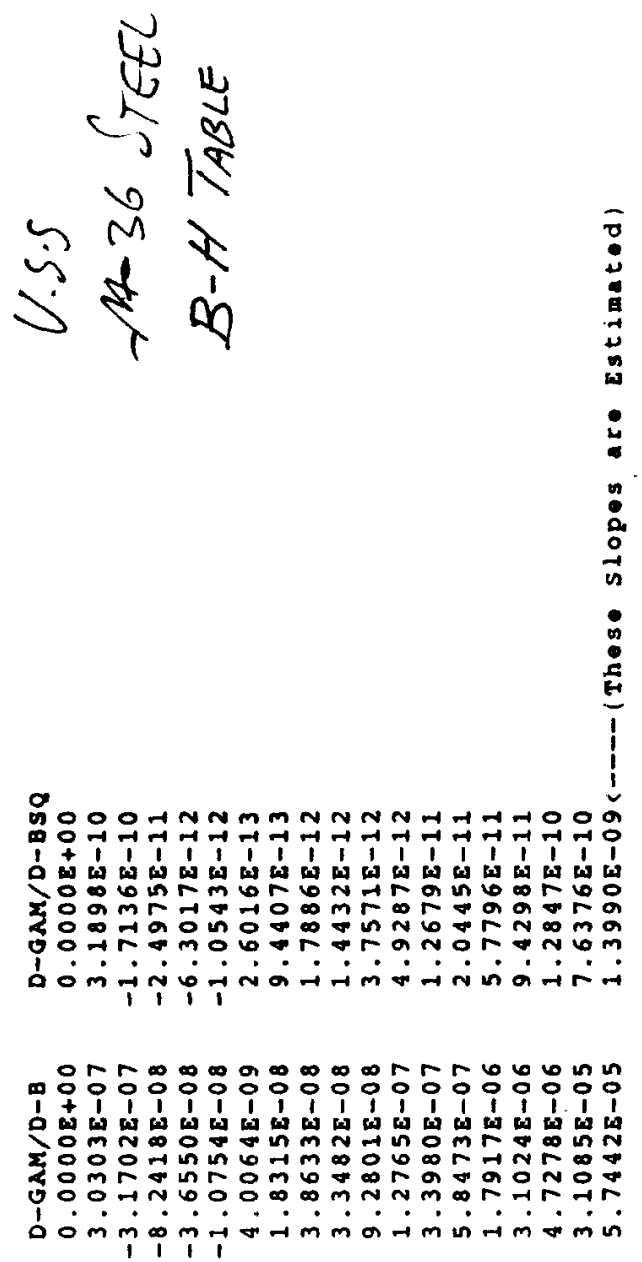

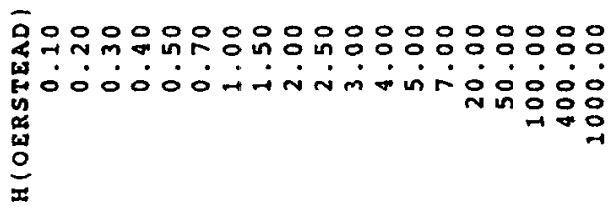

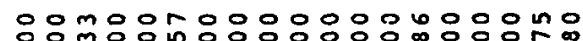

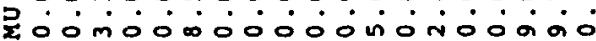

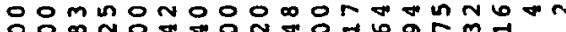

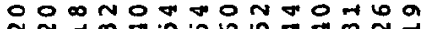

응요

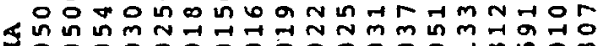

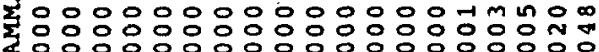

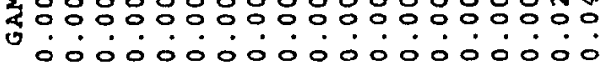

등ㅇㅇㅇㅇㅇㅇㅇㅇㅇㅇㅇㅇㅇㅇㅇㅇㅇㅇㅇㅇㅇㅇㅇㅇㅇㅇㅇㅇㅇㅇㅇㅇㅇ 

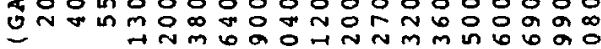
ब

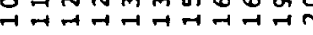

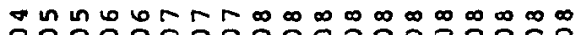

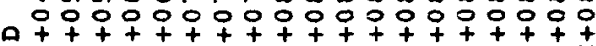

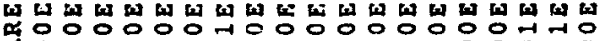

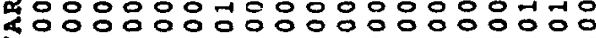

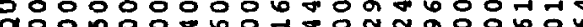

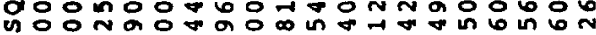
10060.0. z HNm 


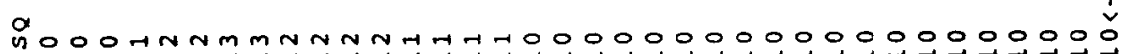

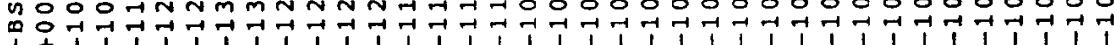

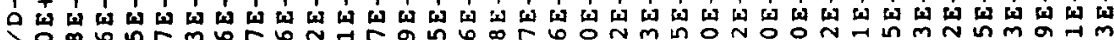
이의

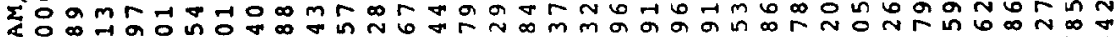

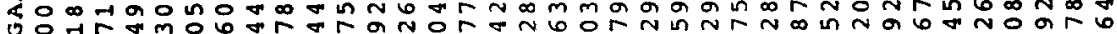

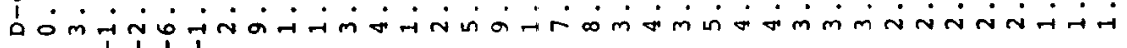

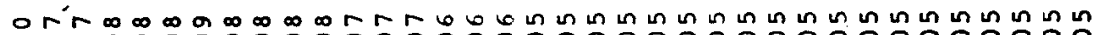

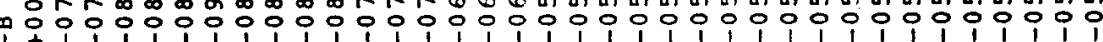
1 t $\begin{aligned} & 1 \\ & 1\end{aligned}$

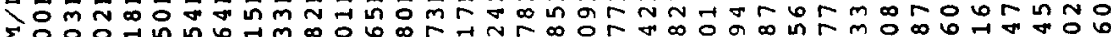

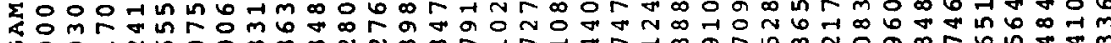

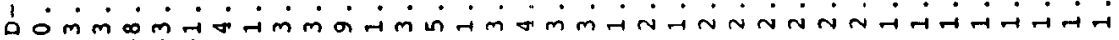
îi

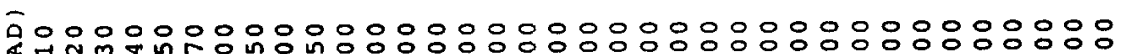

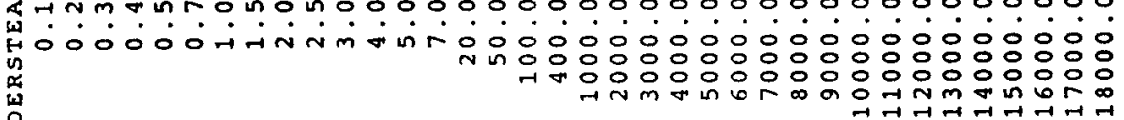
$\underline{0}$

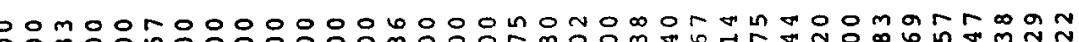

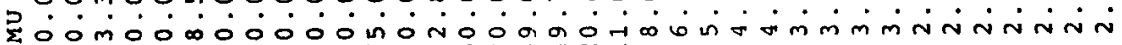

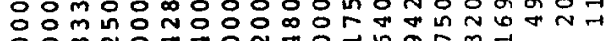

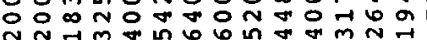

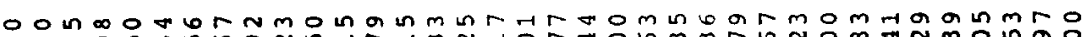

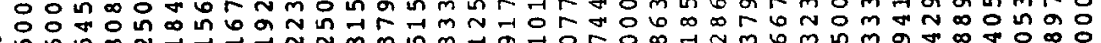

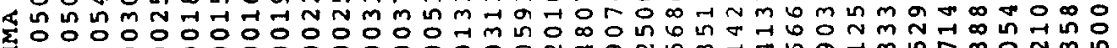

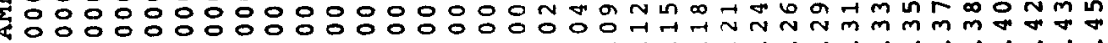

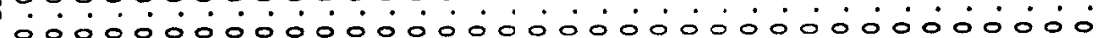

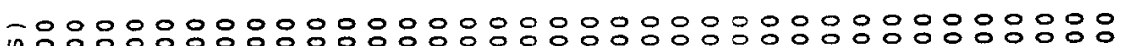

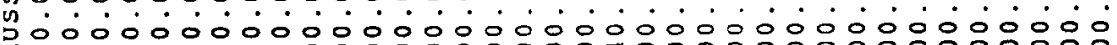

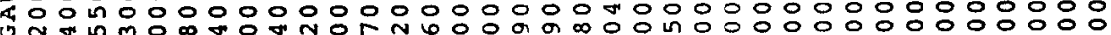

J m

in

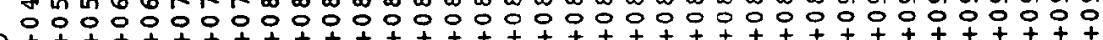

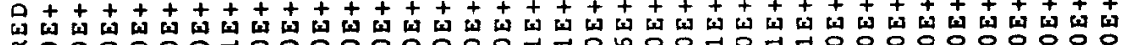

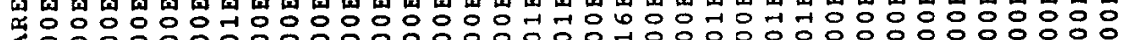

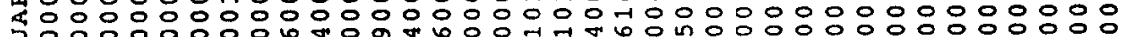

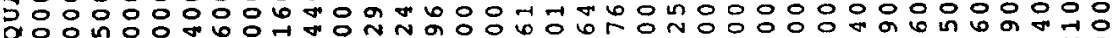

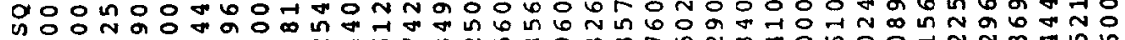

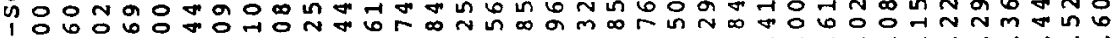

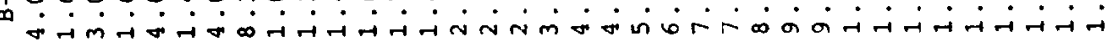
z $\rightarrow$ N 


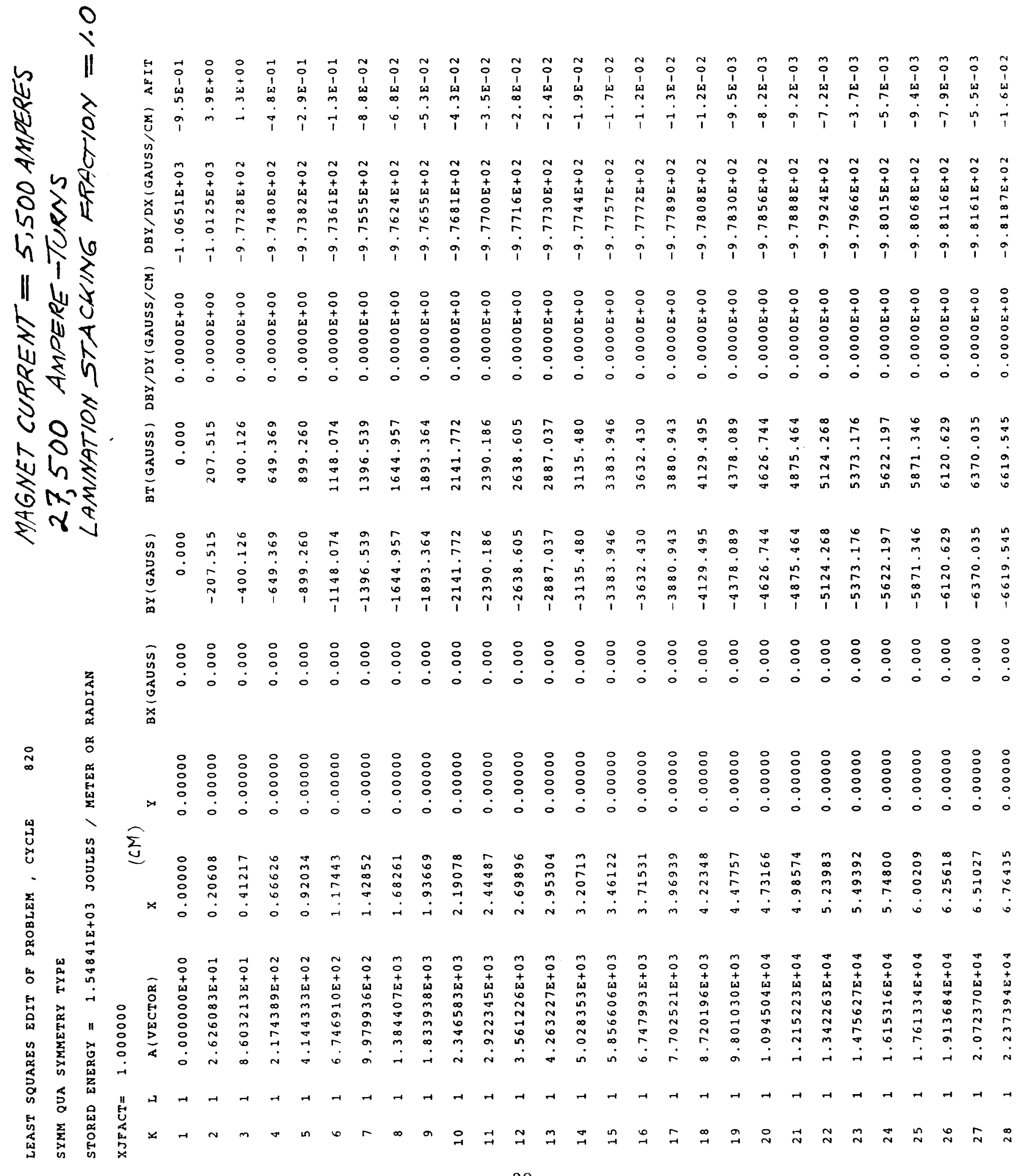




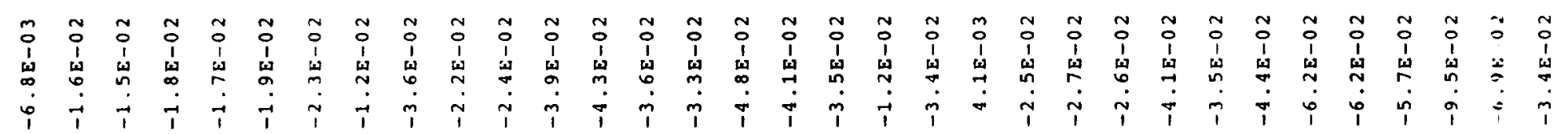

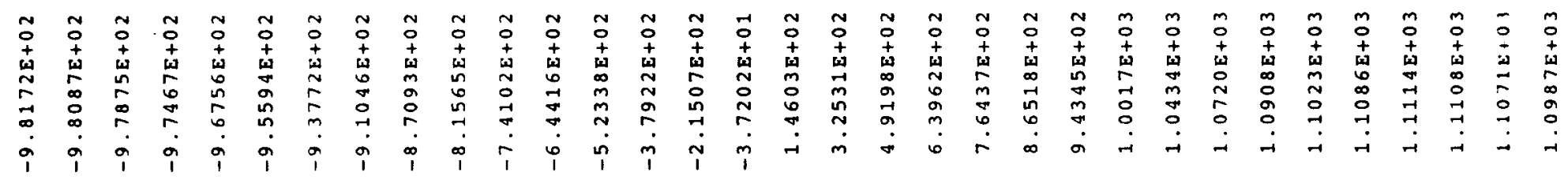

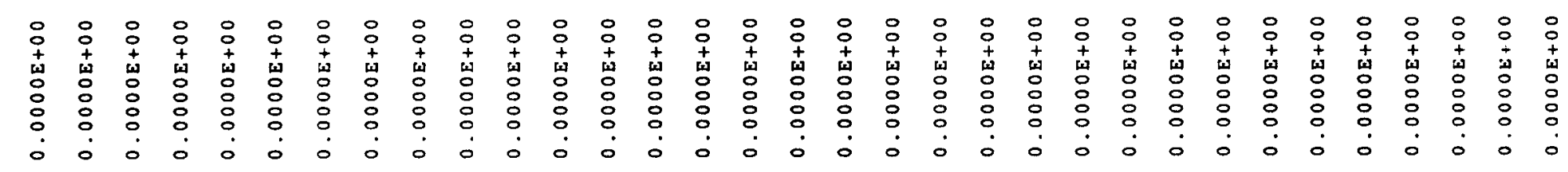

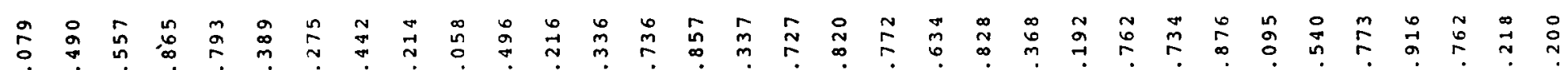

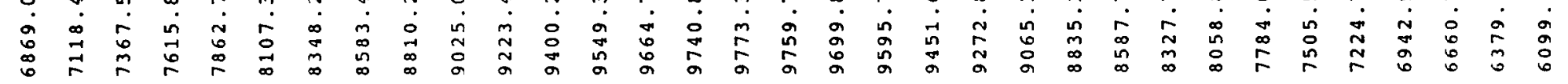

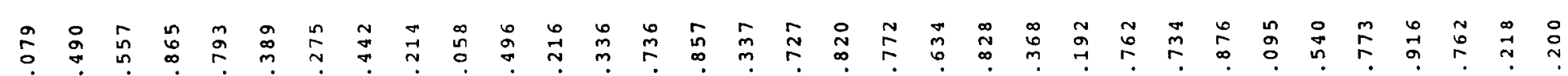

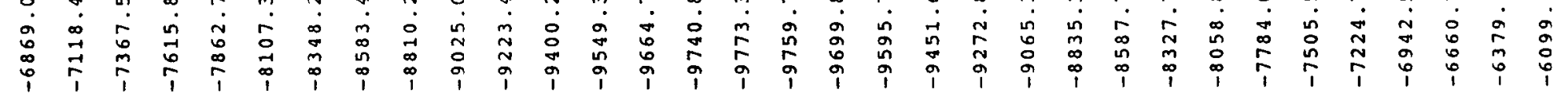

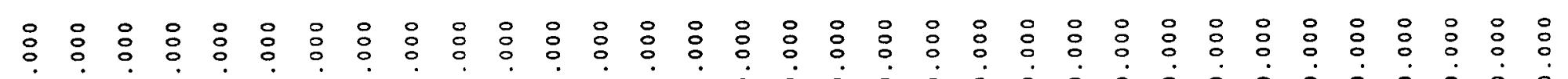

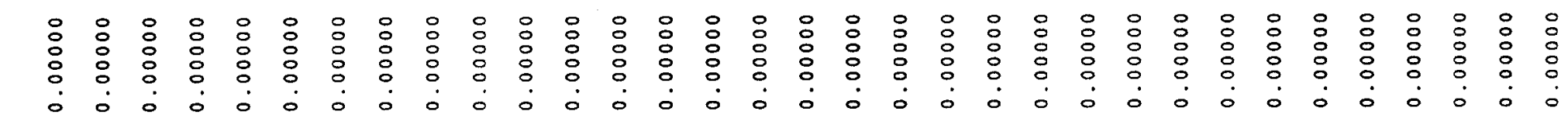

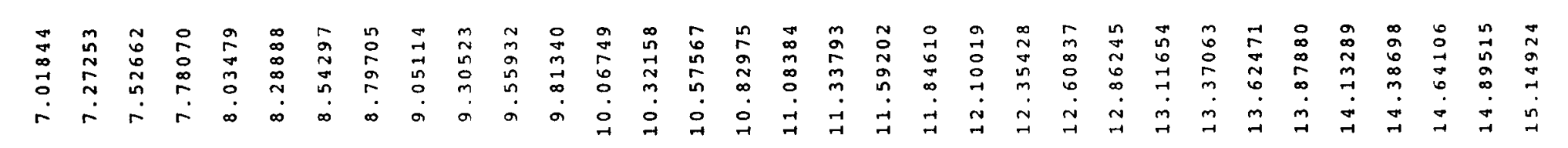

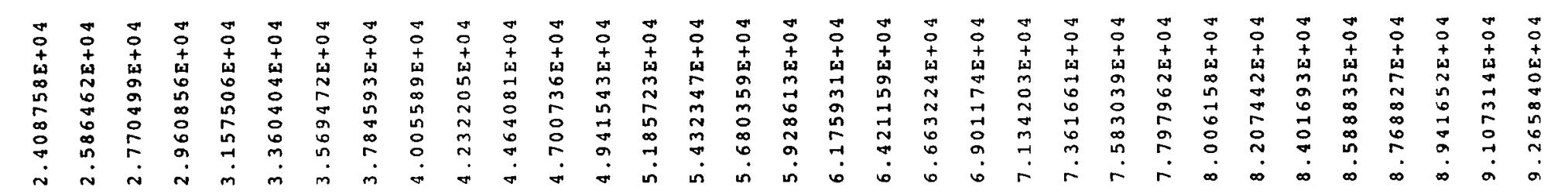

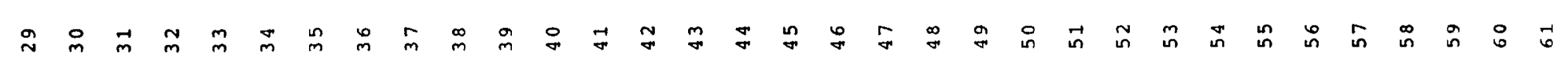



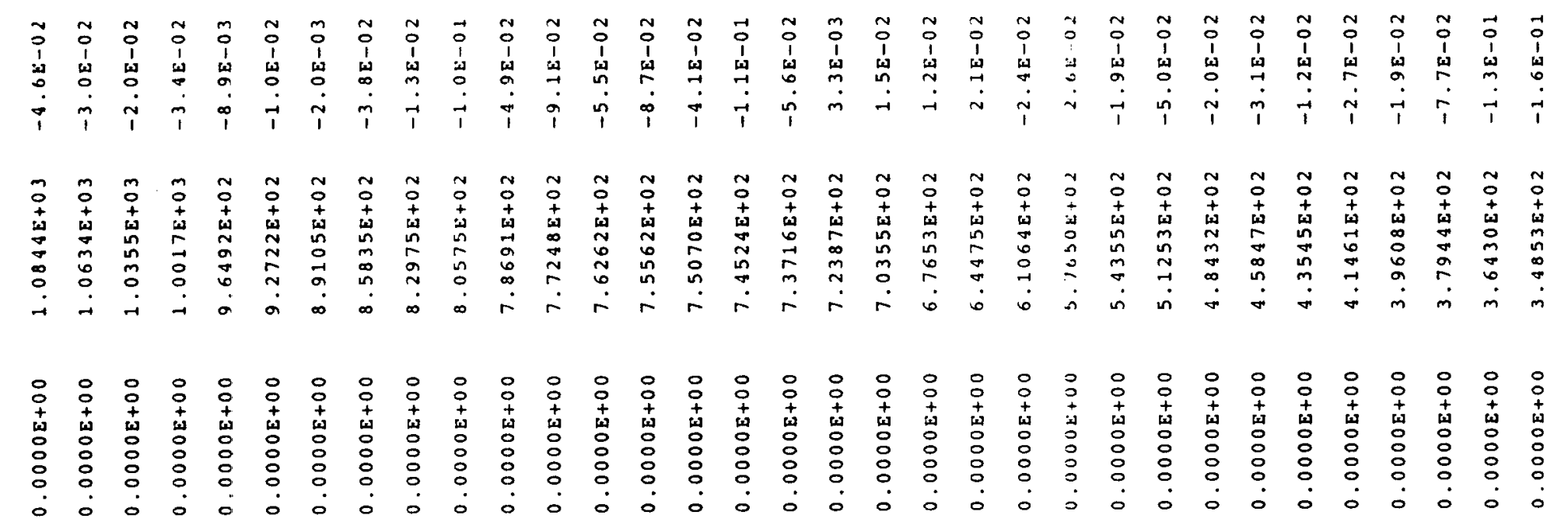

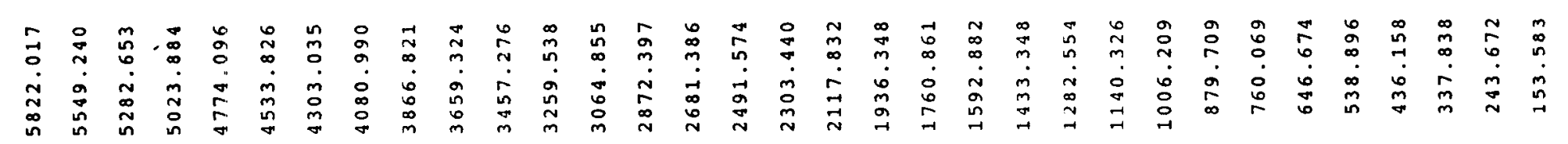

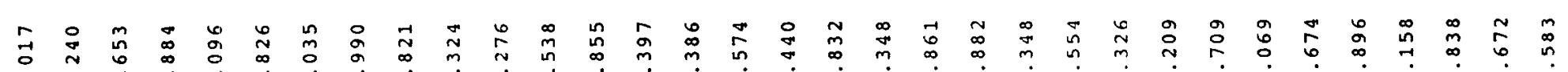

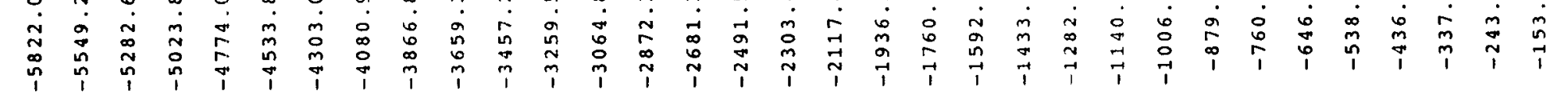

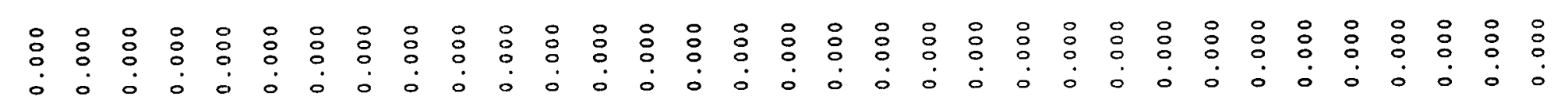

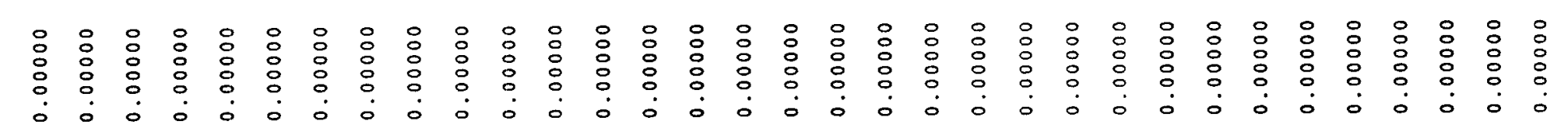

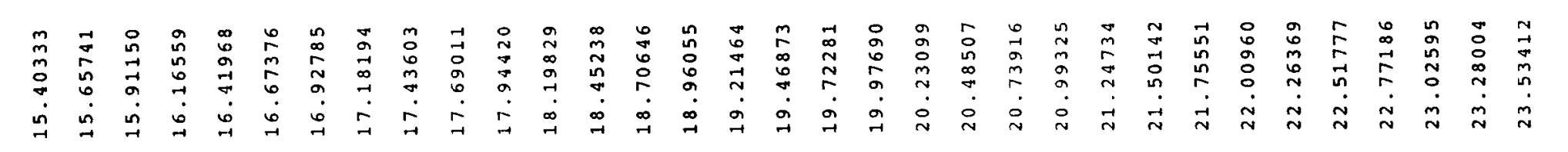

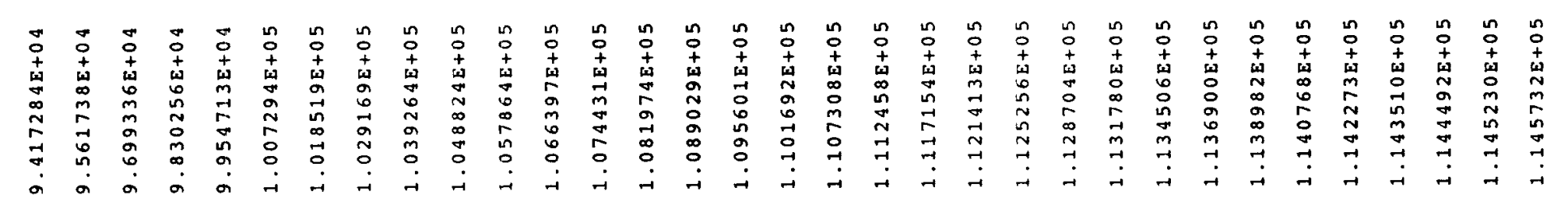

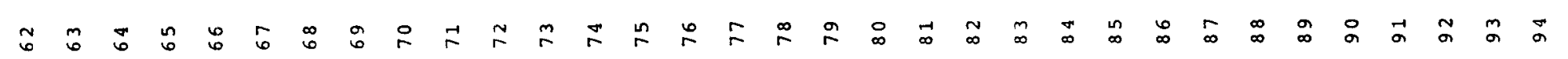

